\title{
Electroencephalographic and clinicopathological studies on Creutzfeldt-Jakob syndrome
}

\author{
K. GOTO, H. UMEZAKI, AND M. SUETSUGU \\ From the Division of Neurology, Kyushu Kosei-Nenskin Hospital, Kitakyushu, and the \\ Department of Psychiatry, Kyushu University School of Medicine, Fukuoka, Japan
}

SYNOPSIS The correlation between the appearance of the characteristic electroencephalographic abnormality, cardinal clinical manifestations, and neuropathological features was studied in four cases of Creutzfeldt-Jakob syndrome consisting of subacute spongiform encephalopathy and classical Creutzfeldt-Jakob disease. Consideration is given to the differences in the electroencephalographic findings between these two subgroups of the syndrome and on the underlying pathological mechanism.

Since Jones and Nevin first described the electroencephalographic (EEG) abnormalities of CreutzfeldtJakob syndrome in 1954 , the periodic, generalised, and three to four phased complexes of sharp and slow waves (periodic synchronous discharges) have been regarded as the most useful clue to the diagnosis of this syndrome. However, the reported incidence of the periodic synchronous discharges in the EEG of patients with this syndrome has varied from 50 to $90 \%$ and the electroencephalographic, clinical, and neuropathological correlation in this syndrome has not been established. The purpose of this report is to compare the appearance of the periodic synchronous discharges with cardinal clinical features and the pathological findings in the cases with CreutzfeldtJakob syndrome.

\section{METHODS}

Four cases with Creutzfeldt-Jakob syndrome verified by necropsy were studied. The age of these patients at the onset of the illness ranged from 57 to 65 years. The EEG were recorded with the Nihonkoden Electroencephalograph type ME $135 \mathrm{D}$ with the 10-20 system of electrode placements. The histograms of the intervals between the periodic synchronous discharges for six minutes were made in each case, and the periodicity, persistency, and fluctuation in the appearance of these discharges were serially analysed. Continuous recording of the EEG, electrooculogram (EOG), electromyogram (EMG), respiratory movements, and electrocardiogram during night sleep were made by

(Accepted 14 May 1976.) the usual methods on one patient in the late stage of the disease.

All necropsy material obtained from the various lobes of the brain, basal ganglia, midbrain, pons, medulla oblongata, cerebellum, and spinal cord were processed according to the standard techniques of neuropathological examination. They were topographically evaluated for the presence and the degree of status spongiosus, astrogliosis, and neuronal loss.

\section{CASE 1}

T. E. After an upper respiratory infection, this 60 year old woman developed rapidly progressive visual loss and disturbance in consciousness. Two months after the onset she developed akinetic mutism.

Fifteen serial EEG recordings were made throughout the six months of the illness. In the first EEG (Fig. 1), recorded when slight disorientation developed, many predominantly anterior high voltage slow waves in the theta range and paroxysmal bursts of delta activity were observed. The rhythmic alpha waves were suppressed and disorganised. In the second EEG (Fig. 2), recorded one week later when the disturbance in consciousness was moderate and transient right sided hemiparesis was observed, bilateral anterior paroxysmal high voltage delta waves increased and the alpha rhythm could not be traced. Slow waves of delta range predominated in the left hemisphere with transient periodic discharges. Two weeks later, she developed akinetic mutism with frequent myolonus, Gegenhalten (resistance against passive movement) and a startle response. The EEG showed diffuse high voltage periodic 


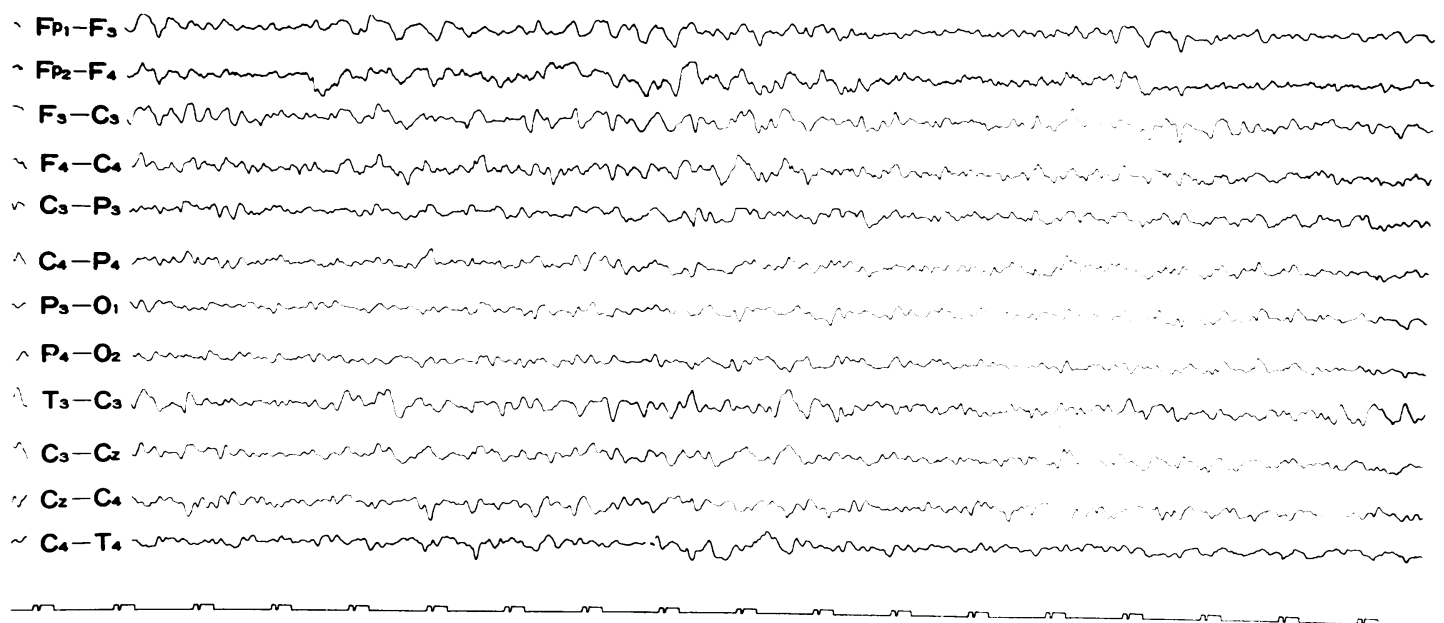

\section{$2 \cdot 14 \cdot 73$}

FIG. 1 Case 1. Diffuse slowing with paroxysmal delta bursts predominantly in the anterior part.

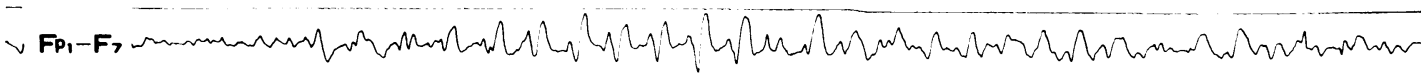
$\sum F_{7}-T_{3}-2$.

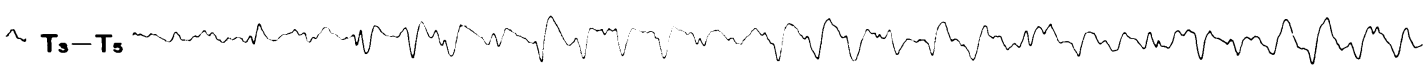
ヘ Ts-O, n

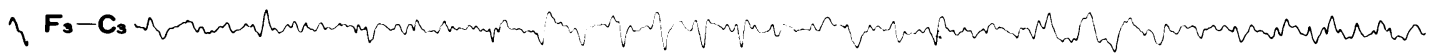
ヘ $\mathbf{C}_{3}-\mathbf{P}_{\mathbf{3}}$

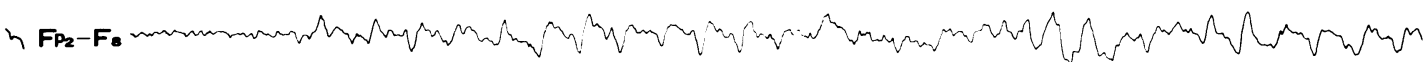

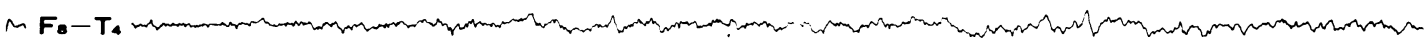

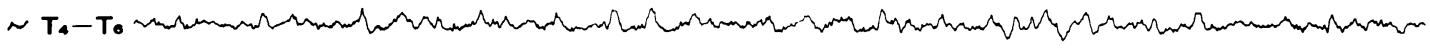

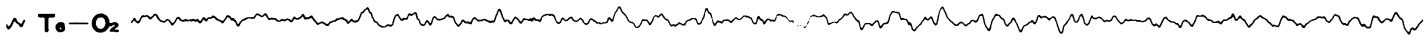

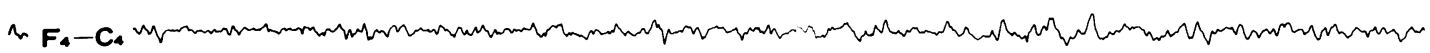

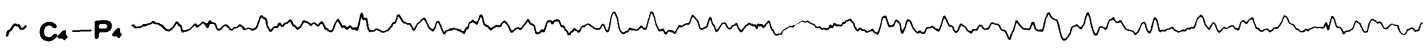

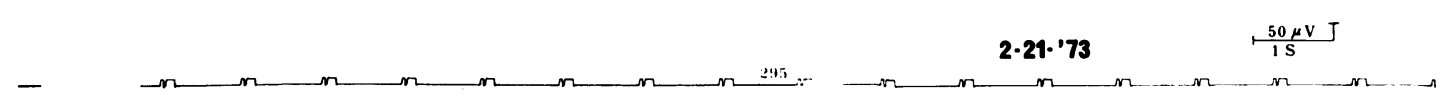

FIG. 2 Case 1. Transient appearance of periodic synchronous discharge predominantly in the left hemisphere. 
synchronous discharges (Fig. 3) and no further change until her death, except for the gradual flattening of the interval tracing between the spikes.

The neuropathological examination revealed diffuse astrogliosis and status spongiosus in the cerebral cortex (Figs. 4 and 5). In comparison with these changes, the degree of neuronal loss was relatively slight. These pathological processes were most prominent in the calcarine cortex of the occipital lobe, less so in the parietal lobe, and far less prominent in the temporal and frontal lobes. The cerebral white matter was unremarkable. In the basal ganglia, status spongiosus was not so prominent, but moderate astrogliosis was observed in the corpus striatum and pulvinar of the thalamus. Slight astrogliosis was also seen in the medial and lateral nuclei of the thalamus and the hypothalamus. In the cerebellum, slight degradation and astrogliosis of the granular cell layer were observed in the dorsal aspect of the hemispheres. The mesencephalon, pons, medulla oblongata and spinal cord were unremarkable.

CASE 2

$T$. $H$. The condition of this 59 year old man progressed from initial symptoms of easy fatigability, insomnia, and visual disturbance to a state of akinetic mutism in 10 weeks.

Four serial EEG recordings were made during the patient's four month course. In the first EEG, recorded when visual disturbance was first noted, the appearance of a small amount of moderate voltage slow waves of theta range was observed diffusely. The occurrence of high voltage polymorphous delta waves was also noted predominantly in the right frontal area. The second EEG, recorded four weeks later when moderate disturbance in consciousness had progressed, showed a further increase of slow waves. The background activity consisted of $8-9 \mathrm{~Hz}$ alpha waves and high voltage theta waves which appeared diffusely and continuously. The high voltage polymorphous delta waves were observed paroxysmally and predominantly in the left hemisphere in this record. In the third EEG, recorded three weeks later when the patient was an akinetic mute with myoclonus, Gegenhalten and a startle response, the periodic synchronous discharges appeared with highest amplitude in the left frontal area (Fig. 6). In the last EEG, recorded three weeks later and one week before death, no focal predominance of the periodic synchronous discharges could be detected.

Neuropathological examination revealed mod-

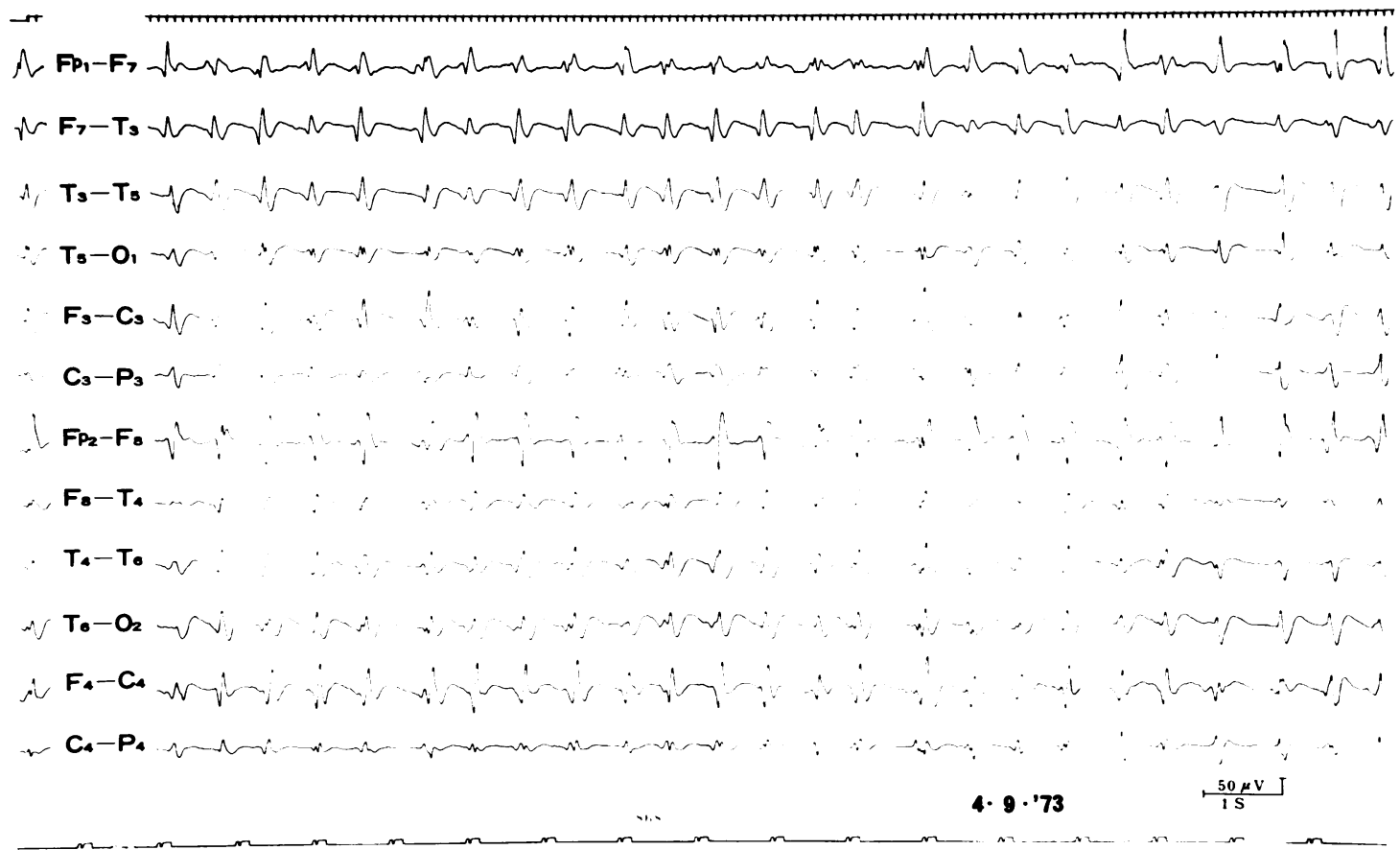

FIG. 3 Case 1. Fully established periodic synchronous discharge. 


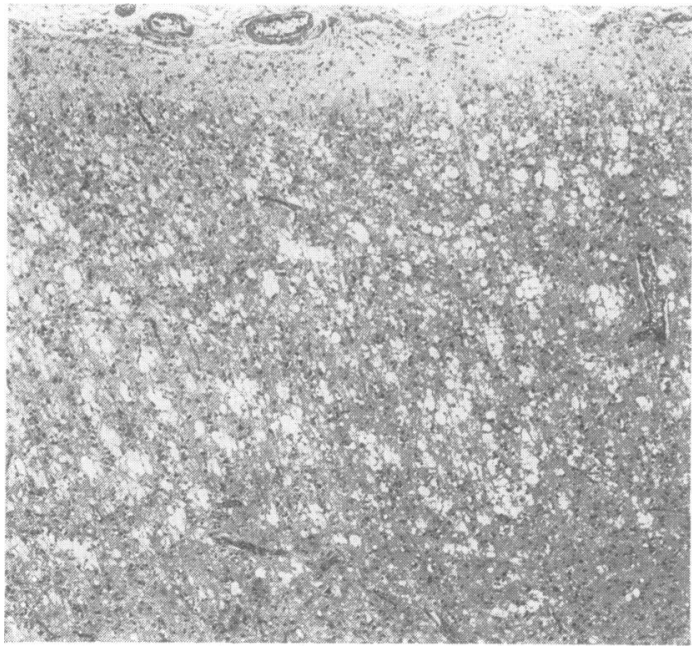

FIG. 4 Case 1. Typical status spongiosus in the superior parietal lobe. Hand $E, \times 41$.

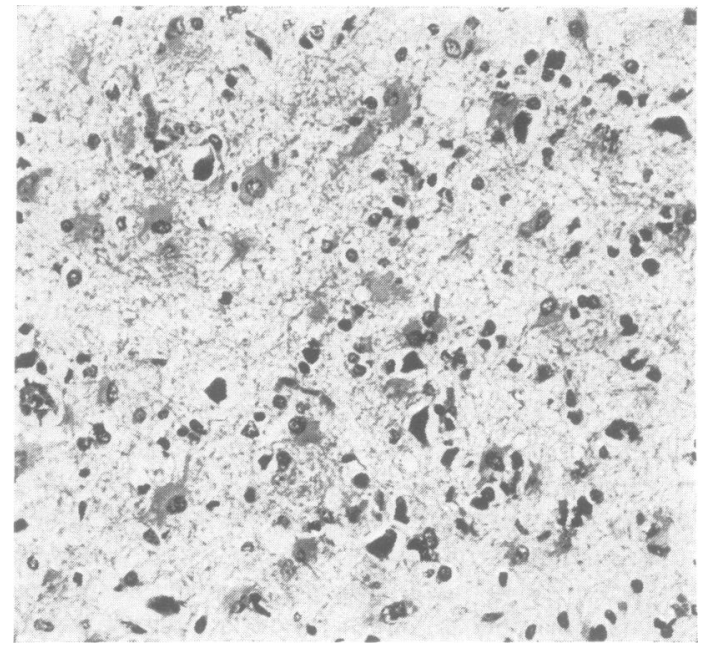

FIG. 5 Case 1. Gemistocytic astrogliosis with neuronal shrinkage in the paracalcarine area. $H$ and $E, \times 212$.

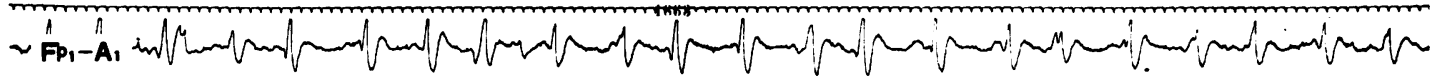

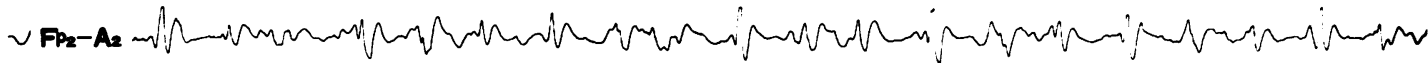

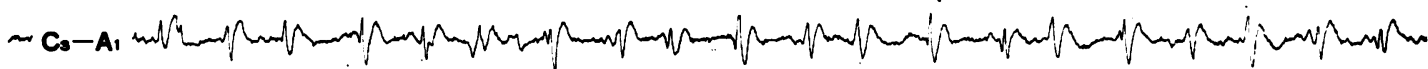

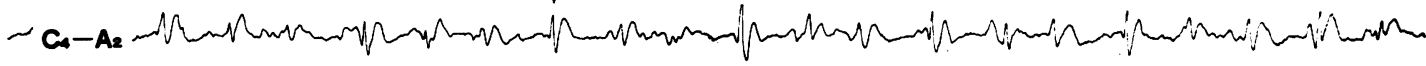

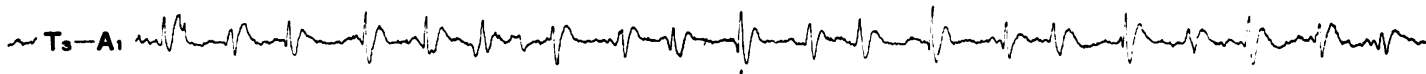

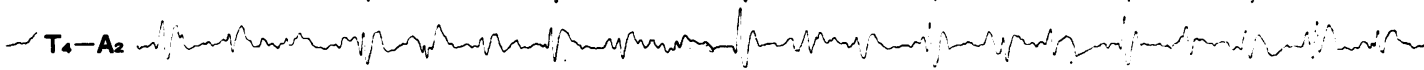

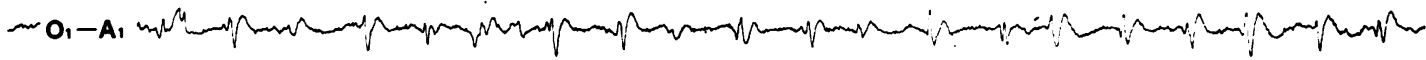

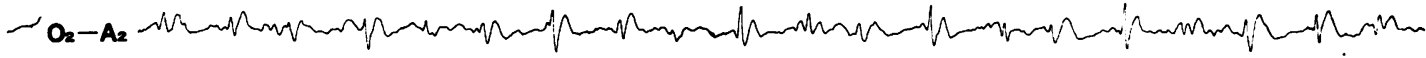

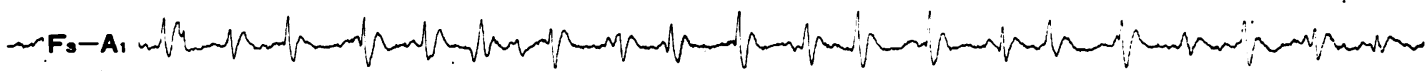

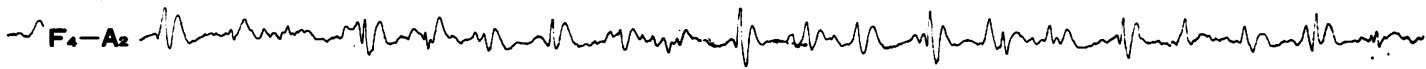

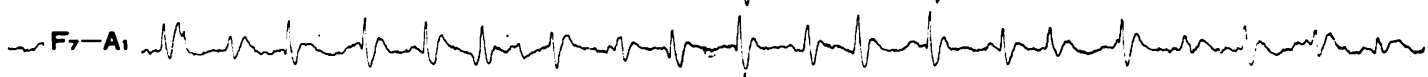

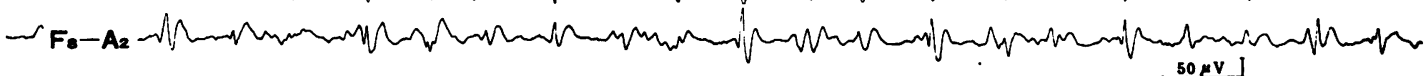
$\frac{50 \mu \mathrm{V}}{1 \mathrm{~s}}$

FIG. 6 Case 2. The amplitude of the periodic synchronous discharge was highest in the left frontal area. 
erate status spongiosus and astrogliosis in the paracalcarine and calcarine cortices of the occipital lobe. These findings were less prominent in the temporal and parietal lobes, and far less prominent in the frontal lobes. Neuronal loss was also notable in the area where the status spongiosus was prominent. Most of the remaining neurones showed a disturbance in arrangement and various aspects of degeneration, but many normal neurones were found. The cerebral white matter was unremarkable. There was no astrogliosis, status spongiosus and neuronal loss or degeneration in the basal ganglia. The brain-stem, the cerebellum, and the spinal cord were unremarkable.

\section{CASE 3}

$M . O$. After four weeks' history of frequent headaches and dizzy spells, metamorphopsia occurred in this 66 year old man. Disturbance in consciousness progressed rapidly into akinetic mutism within seven weeks. He died of massive haemorrhage from a gastric ulcer three months after the occurrence of his initial symptoms.

In the first EEG, recorded when the patient became delirious, moderate to high voltage slow waves in the theta range were observed diffusely and sporadically, but some alpha waves still remained. In the second EEG (Fig. 7), recorded one week later when myoclonus, rigidity, and akinetic mutism had developed, periodic synchronous discharges appeared diffusely and continuously. The third EEG, recorded three weeks later and two weeks before his death, showed no essential change except for the disappearance of rhythmic waves in the background activity compared with the previous examination.

Neuropathological examination of the brain revealed moderate astrogliosis and slight status spongiosus in the calcarine cortex of the occipital lobe. Much less astrogliosis was noted in the frontal, temporal, parahippocampal, and precentral gyri. Slight gliosis was found in the pulvinar of thalamus. The brain stem and the cerebellum were unremarkable.

\section{CASE 4}

$S . M$. This 56 year old woman showed slowly progressive mental deterioration which led to akinetic mutism within nine months.

Seven serial EEG recordings were performed over the 15 month duration of the illness. The basic pattern of the first EEG, recorded six months after the

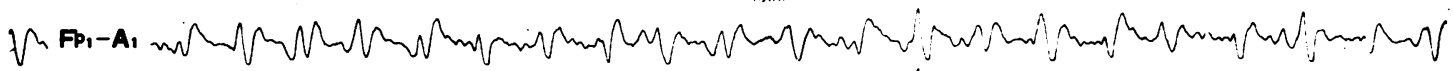

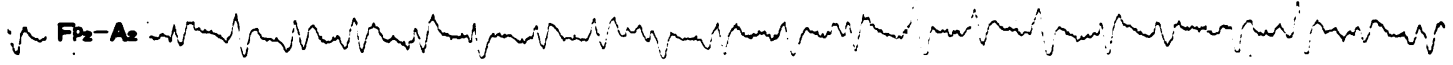

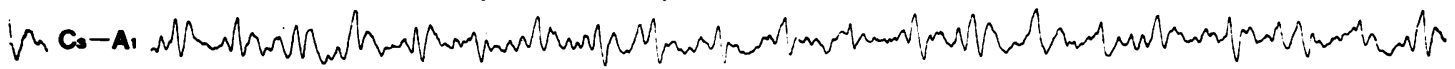

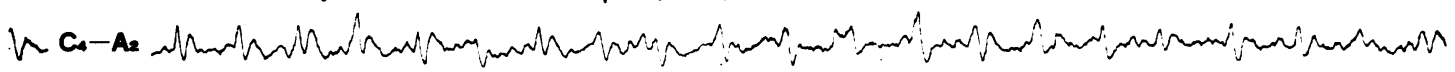

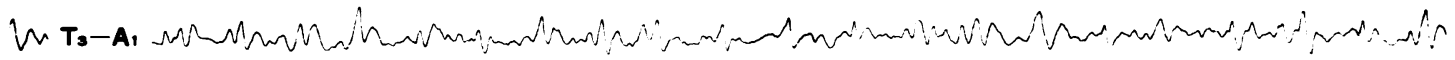

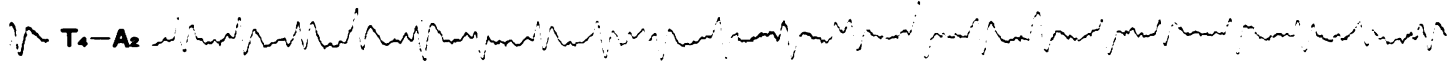

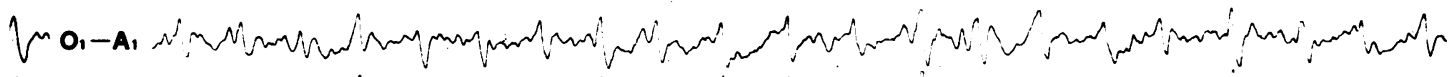

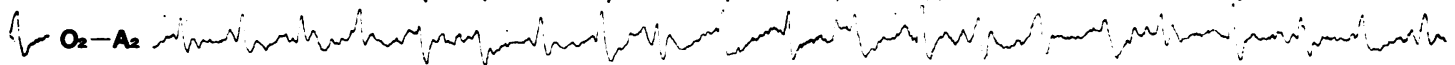

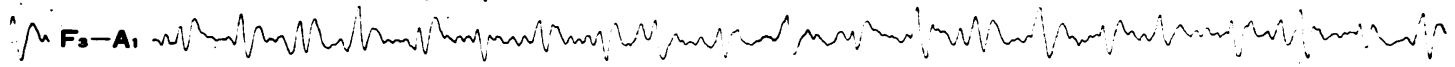

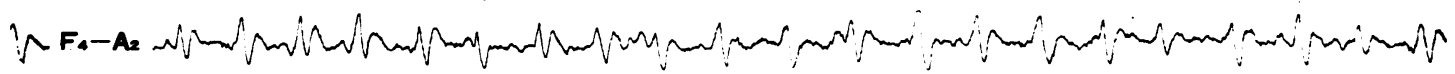

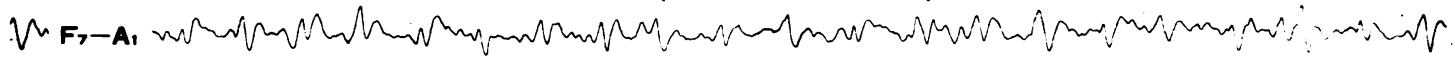

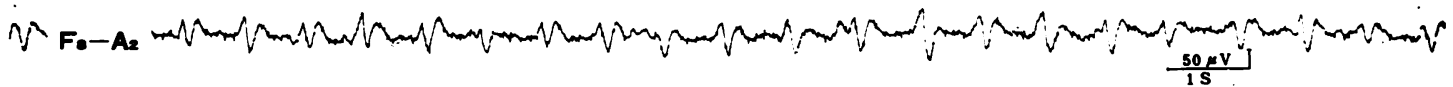
(C)

FIG. 7 Case 3. Periodic synchronous discharge with small amount of rhythmic wave in the background activity. 
onset when dementia was already severe, consisted of moderately high voltage, rhythmic activity of theta range with a small amount of alpha waves. A moderate amount of high voltage, irregular delta waves of 1 to $3 \mathrm{~Hz}$ range appeared sporadically and diffusely. The second EEG, recorded two months later, when generalised hyperreflexia and bilateral Babinski signs were observed, revealed a diffuse increase in the high voltage delta waves and the disappearance of the alpha rhythm. Rarely, high voltage spikes, sometimes triphasic, appeared diffusely and sporadically. The third EEG, recorded one month after the previous examination, showed periodic synchronous discharges for the first time (Fig. 8). Clinically, then, she was an akinetic mute with marked rigidity of her extremities and Gegenhalten. The shape of the periodic synchronous discharges, however, was variable and their appearance was intermittent. The background activity was composed of high voltage irregular theta and delta waves. In the EEG recorded only eight days later, periodic synchronous discharges could not be detected. Bilaterally synchronous high voltage sharp and slow wave complexes were scattered diffusely but without obvious periodicity. The following EEG showed a gradual decline in the amplitude of the slow waves. The last EEG, recorded two months before her death showed a relatively simple pattern consisting of moderate voltage, irregular slow waves of theta range alternating with an almost flat pattern.

The histopathological examination revealed diffuse gliosis and status spongiosus of the cerebral cortex. These findings were most prominent in the frontal lobe, less so in the temporal and parietal lobes, and slight in the occipital lobe. Neuronal loss was relatively slight. In the cerebral white matter, slight pallor of myelin sheaths was observed. Slight astrogliosis was present in the basal ganglia, the mesencephalon, the central grey matter, and the red nucleus without neuronal loss and status spongiosus. In the cerebellar cortex, focal areas of astrogliosis and status spongiosus of slight degree were found. Small foci showing ischaemic changes in Purkinje cells were noted. The spinal cord was unremarkable.

The early EEG changes in our four cases were progressive increase in slow waves and gradual disappearance of the background alpha waves. Most of the slow waves initially noted were in slow theta range of moderate to high voltage and, with the progression of the disturbance in consciousness or dementia, the high voltage irregular delta waves increased in

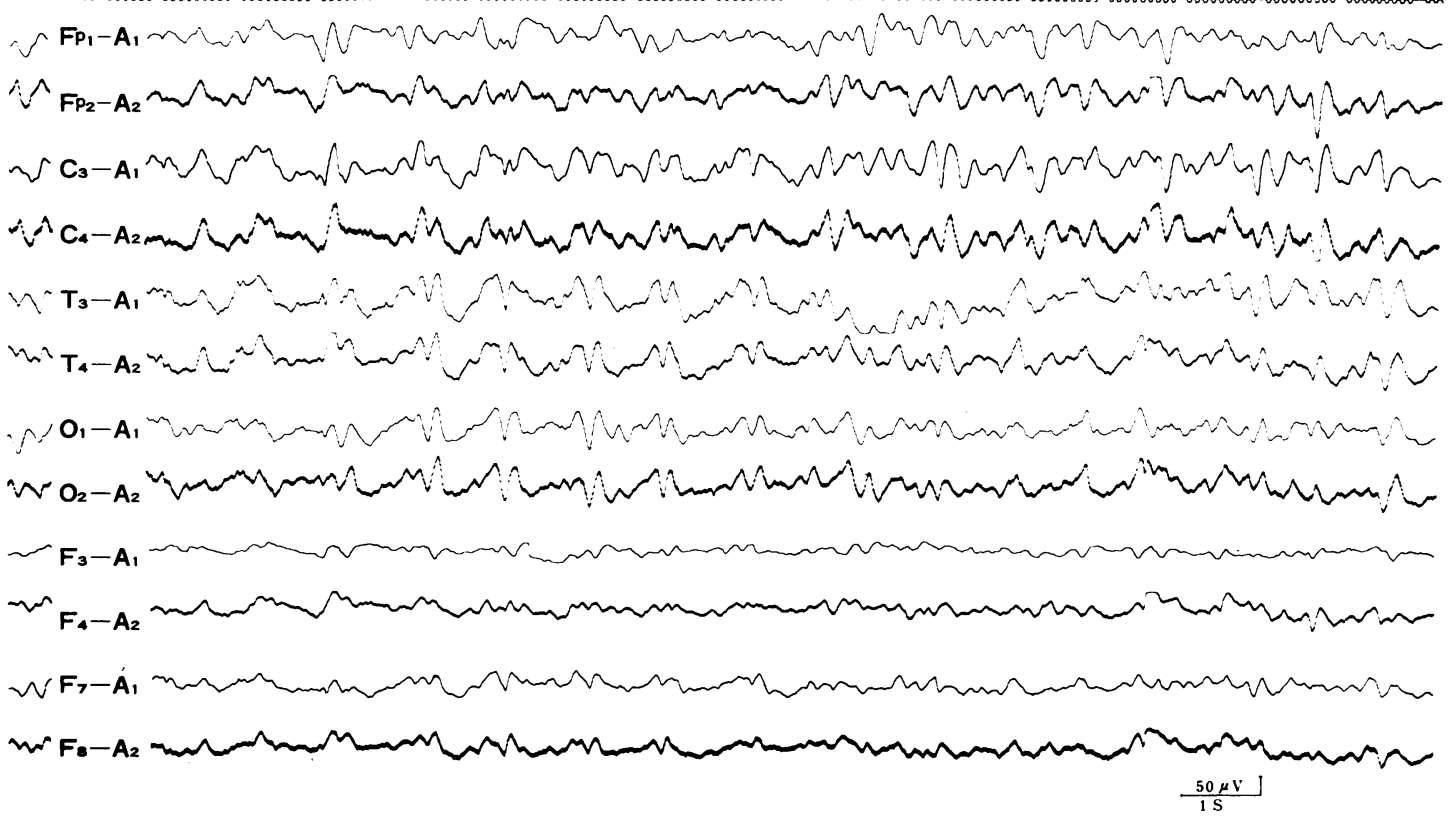

FIG. 8 Case 4. Appearance of periodic synchronous discharge was observed in this record alone among the seven serial records. 
frequency. Generally, the distribution of the slow waves was diffuse, but focal predominance was observed transiently in cases 1,2 , and 3 , in the early stage. Periodic synchronous discharges were recorded in all four cases. In cases 1,2 , and 3, they were recorded for the first time seven to 11 weeks after the onset of the initial symptom, and did not disappear until shortly before each patient's death. Initially, the periodic synchronous discharges were not constant, exhibiting a variable interval and focal predominance, but rapidly the discharges became continuous, highly periodic, generalised, and bilaterally synchronous. These features were clearly demonstrated by plotting a serial interval histogram of the periodic synchronous discharges (Fig. 9), which also showed that, when the continuity of the discharges was established, their periodicity was markedly stable with a dominant interval of $0.6 \mathrm{~s}$. $(1.7 \mathrm{~Hz})$ in cases 1,2 , and 3 (Fig. 10). In addition, the interval between the discharges became slightly longer toward the terminal stage in cases 1 and 2 . In contrast with these cases, periodic synchronous discharges appeared in only one EEG recorded in the late stage in nine serial recordings of case 4 . The mode of appearance of the periodic synchronous discharges in this patient was intermittent and the period of the discharges was longer and less constant than the other three cases. The wave forms of the periodic synchronous discharges were similar in cases 1,2 , and 3 . They were high voltage complexes composed of a spike or a sharp wave followed by a slow wave. The periodically recurring complex of case 4 was a high voltage irregular slow wave. Its duration was 0.4 to 0.5 seconds in cases 1,2 , and 3 , and 0.7 to 0.8 seconds in case 4 .

A close correlation between the occurrence of periodic synchronous discharges and progression of disturbed consciousness, myoclonus, rigidity, Gegenhalten, and startle response was found clinically in our cases. After the mode of appearance of the spontaneous discharge became continuous, its rate was not influenced by exogenous stimuli, but by an endogenous factor, probably sleep. To confirm these observations, an overnight EEG was recorded in case 1 (Fig. 11). Periodic synchronous discharges and myoclonus were observed continuously for several hours after the EEG recording was started. Then the myoclonus ceased and the periodic discharges disappeared completely for episodes of several minutes. This phase (named phase II for convenience) appeared repeatedly until daybreak. In phase II, moderate voltage rhythmic activity of 5 to $8 \mathrm{~Hz}$ appeared diffusely in place of the periodic discharges. The patient's neck and extremities were flaccid, without Gegenhalten. Respiration and heart rate were slow and regular. No rapid eye movements could be observed in the EOG. At the end of this phase, the
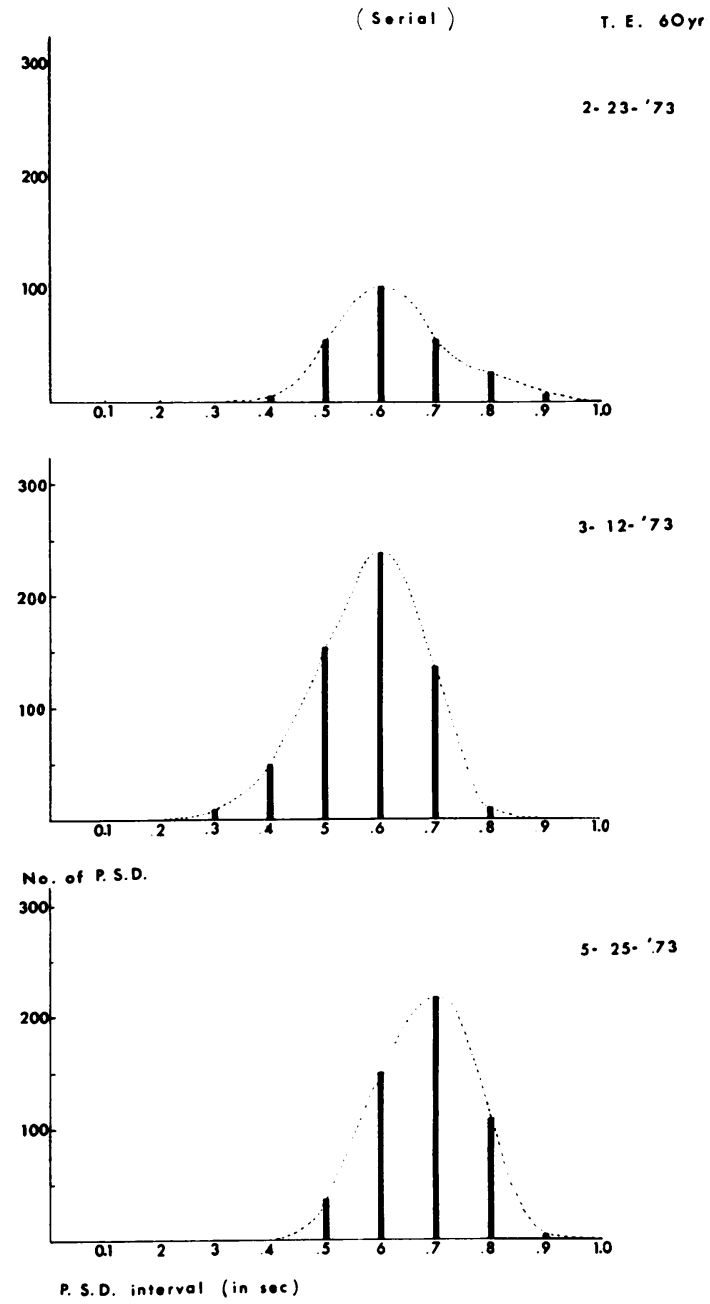

FIG. 9 Case 1. Interval histograms of periodic spontaneous discharge for six minutes in various stages.

rhythmic waves of the theta band disappeared suddenly and polyphasic, high voltage slow waves appeared intermittently with concomitant myoclonus. Initially, the period of these slow waves was relatively long, but gradually the initial component of the high voltage slow waves became sharp and the frequency shortened to $1.7 \mathrm{~Hz}$. Associated with these changes, myoclonus became violent, and one to one correspondence with the periodic synchronous discharge was lost. At the same time, muscular rigidity increased and respiratory movement became fast and irregular. No normal sleep pattern could be observed. 

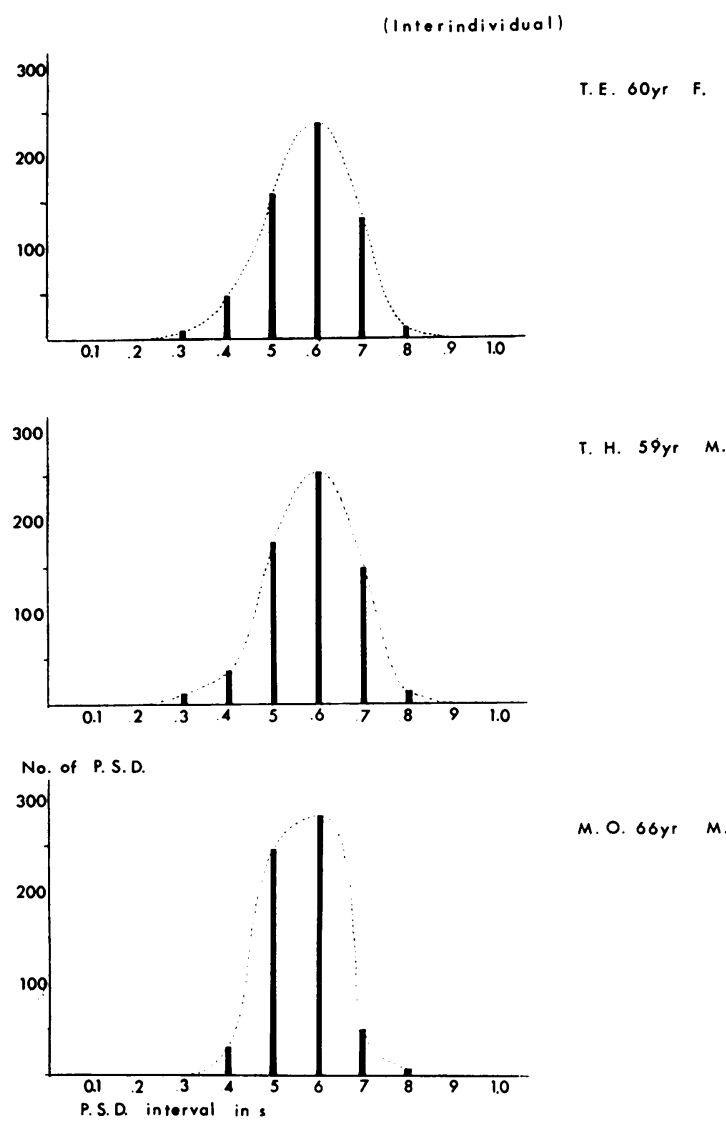

FIG. 10 Cases 1, 2, 3. Interval histograms of periodic spontaneous discharges for six minutes when they were fully established.

\section{DISCUSSION}

Increased attention has recently been given to the diagnostic value of the EEG in the Creutzfeldt-Jakob syndrome. Slowing of the background activity, the most common finding in reported cases, is not a specific finding. The progressive degradation of background activity which finally leads to the appearance of periodic spontaneous discharge has been observed by several authors (Burger et al., 1972; Goldhammer et al., 1972; Lee and Blair, 1973; Elliott et al., 1974). Though the periodic discharge has been recorded in various pathological conditions of the brain (Cobb and Hill, 1950; Lesse et al., 1958; Hoefer et al., 1963; Fenyö and Hasznos, 1964; Cobb, 1966; Gloor et al., 1968; Ohya et al., 1974), the Creutzfeldt-Jakob syndrome shows the most characteristic pattern. The distribution is generalised and bilaterally synchro- nous, the periodicity is constant, the wave form is rather uniform consisting of a high voltage spike or sharp and slow wave complex, and its appearance is usually persistent until shortly before death.

Of the four cases of Creutzfeldt-Jakob syndrome presented here, two subgroups could be discerned clinicopathologically: cases 1,2 , and 3 are thought to be the so-called Heidenhain subtype of subacute spongiform encephalopathy and case 4 is considered to be more akin to the classical Creutzfeldt-Jakob disease. It is interesting that there seem to be some remarkable differences in the EEG findings between these two subgroups of the syndrome (Table 1). In reviewing the EEG findings of previously reported cases of subacute spongiform encephalopathy (Gloor et al., 1968; Goldhammer et al., 1972; Burger et al., 1972), it is confirmed that the above mentioned characteristics of the periodic spontaneous discharges of the Creutzfeldt-Jakob syndrome are most marked in subacute spongiform encephalopathy. It is said that the periodic discharge is recorded in about one-half of cases with Creutzfeldt-Jakob syndrome verified by necropsy (May, 1968; Yuasa, 1969; Hamanaka et al., 1970). However, in reports dealing only with subacute spongiform encephalopathy, almost all of the cases showed the periodic synchronous discharges (Goldhammer et al., 1972; Burger et al., 1972; Roos et al., 1973). It may well be said that subacute spongiform encephalopathy, which is regarded as the most clearly definable subtype of Creutzfeldt-Jakob syndrome clinically and neuropathologically (Matsuoka et al., 1970; Schlote, 1970), also has the most characteristic EEG findings.

With regard to previously reported studies (Nelson and Leffman, 1963; Rayport, 1963; Gloor et al., 1968; Lee and Blair, 1973), it may be postulated that the periodic synchronous discharge develops when the loss of cortical inhibition over subcortical structures brings about hypersynchronisation of the neurone group in the central grey matter. Such abnormal discharges are conducted upward by the relatively uninvolved diffuse thalamocortical projection system and fire the remaining cortical neurones. Though there are some reports on a close relationship between the appearance of the periodic EEG complex and respiration and sleep pattern in children with subacute sclerosing leucoencephalitis (Fenyö and Hasznos, 1964; Scollo-Lavizzari, 1968), such a fact has not been reported in the Creutzfeldt-Jakob syndrome. The mode of appearance of the periodic synchronous discharge and the close relationship between it and several physiological aspects which were clearly shown in the transitional phase of the sleep EEG of our case 1 seem to be good evidence of hypersynchronisation of the neurone group in the brain stem reticular formation. The recurrent ap- 


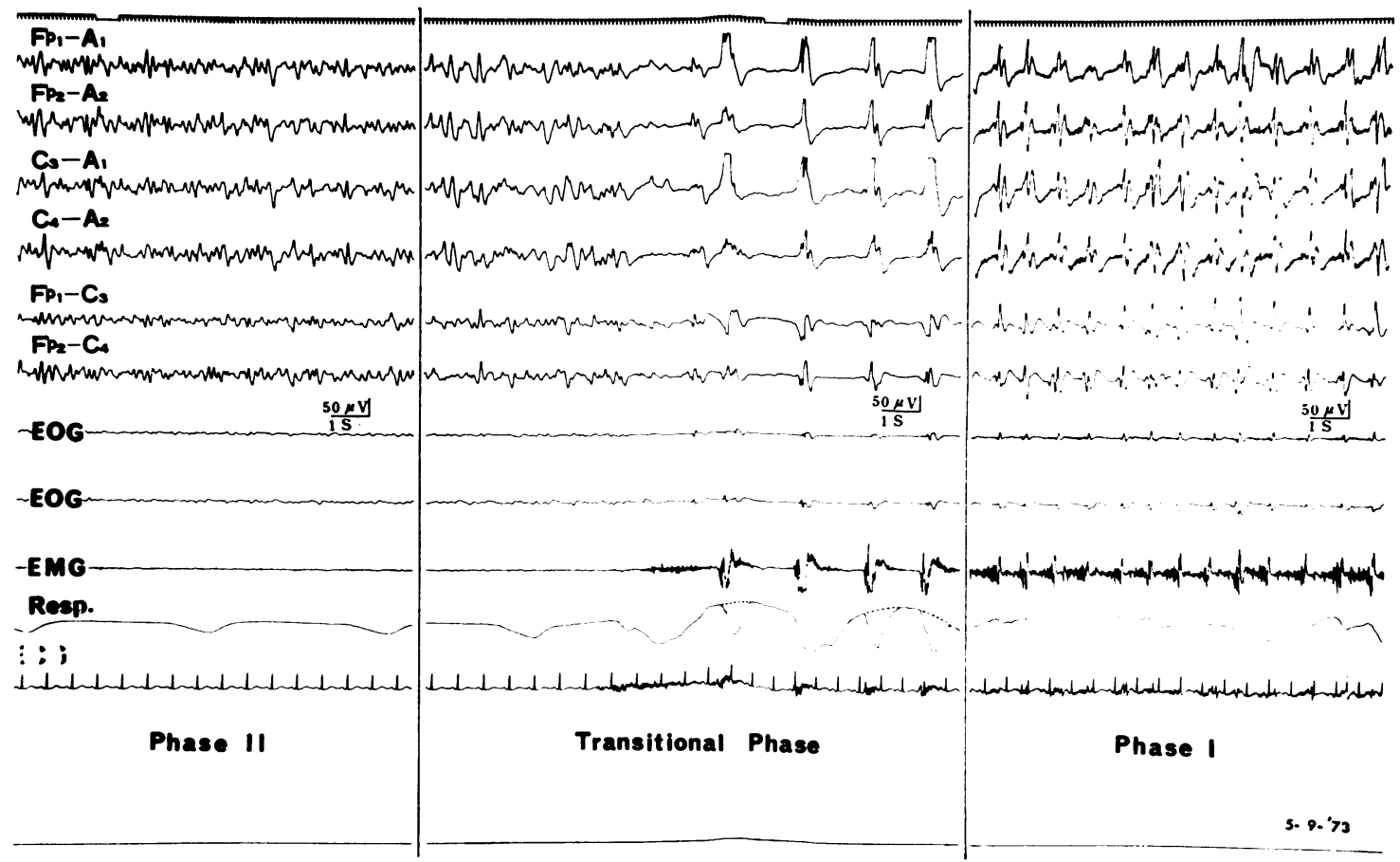

FIG. 11 Case 1. Two different phases of polygram during night sleep. Note the drastic changes in the EMG, respiration and electrocardiogram accompanying the appearance of periodic spontaneous discharge in the transitional phase.

T A B L E 1

SUMMARY OF CLINICAL, NEUROPATHOLOGICAL, AND ELECTROENCEPHALOGRAPHIC FEATURES

Cases 1, 2, and 3

Clinica! features

Initial sign of CNS involvement

Cardinal manifestations

Total duration of illness

Neuropathological findings

Cerebral cortex most severely involved

White matter

Brain stem

Clinicopathological diagnosis

Periodic spontaneous discharge in EEG

Wave form

Duration

Period

Mode of appearance
Cortical blindness

Rapidly progressive disturbance in consciousness

3 to $6 \mathrm{~m}$

Occipital lobe

Uninvolved

Uninvolved

Subacute spongiform encephalopathy

Spike and slow wave complex, stereotyped 0.4 to $0.5 \mathrm{~s}$

$1.7 \mathrm{~Hz}$, stable

Persistent and continuous
Case 4

Mental deterioration

Gradually progressive dementia

$15 \mathrm{~m}$

Frontal lobe

Slightly involved

Slightly involved

Classic Creutzfeldt-Jacob disease

High voltage slow wave, variable 0.7 to $0.8 \mathrm{~s}$

Variable

Transient and intermittent 
pearance of the rhythmic theta waves in phase II of the sleep EEG seems to suggest that the electrical activity of cortical neurones is preserved to some extent even in the later stage. This is consistent with the histological observation which revealed relative preservation of normal cortical neurones in our cases. The characteristic features in the periodic spontaneous discharge of the subacute spongiform encephalopathy group of our cases are considered to be based on the relative preservation of white matter and brain stem structures. The atypical appearance of the periodic spontaneous discharge in case 4 may suggest that marked extension of the disease process occurred in the cephalocaudal direction relatively early in this case.

The authors thank Dr Bruce H. Dobkin, University of California at Los Angeles, who reviewed the manuscript and offered helpful advice.

\section{REFERENCES}

Burger, L. J., Rowan, A. J., and Goldensohn, E. S. (1972). Creutzfeldt-Jakob disease. Archives of Neurology (Chi.), 26, 428-432.

Cobb, W. (1966). The periodic events of subacute sclerosing leucoencephalitis. Electroencephalography and Clinical Neurophysiology, 21, 278-294.

Cobb, W., and Hill, D. (1950). Electroencephalogram in subacute progressive encephalitis. Brain, 73, 392-404.

Elliott, F., Gardner-Thorpe, C., Barwick, D. D., and Foster, J. B. (1974). Jakob-Creutzfeldt disease. Modification of clinical and electroencephalographic activity with methylphenidate and diazepam. Journal of Neurology, Neurosurgery, and Psychiatry, 37, 879-887.

Fenyö, E., and Hasznos, T. (1964). Periodic EEG complexes in subacute panencephalitis: reactivity, response to drugs and respiratory relationships. Electroencephalography and Clinical Neurophysiology, 16, 446-458.

Gloor, P., Kalabey, O., and Giard, N. (1968). The electroencephalogram in diffuse encephalopathies: electroencephalographic correlates of grey, and white matter lesions. Brain, 91, 779-802.

Goldhammer, Y., Bubis, J. J., Sarova-Pinhas, I., and Braham, J. (1972). Subacute spongiform encephalopathy and its relation to Jakob-Creutzfeldt disease: report on six cases. Journal of Neurology, Neurosurgery, and Psychiatry, 35, 1-10.
Hamanaka, T., Kawai, I., and Tatebayashi, Y. (1970). Über Periodizität der Abnormen Entladungen im Elektroenzephalogram. Brain and Nerve, 22, 13-26.

Hoefer, P. F. A., Denapoli, R. A., and Lesse, S. (1963). Periodicity and hypsarrhythmia in the EEG. Archives of Neurology (Chic.), 9, 112-124.

Jones, D. P., and Nevin, S. (1954). Rapidly progressive cerebral degeneration (subacute vascular encephalopathy) with mental disorder, focal disturbances and myoclonic epilepsy. Journal of Neurology, Neurosurgery, and Psychiatry, 17, 148-159.

Lee, R. G., and Blair, R. D. G. (1973). Evolution of EEG and visual evoked response changes in JakobCreutzfeldt disease. Electroencephalography and Clinical Neurophysiology, 35, 133-142.

Lesse, S., Hoefer, P. F. A., and Austin, J. H. (1958). The electroencephalogram in diffuse encephalopathies. Archives of Neurology and Psychiatry (Chic.) 79, 359-375.

Matsuoka, T., Hamanaka, T., Taii, S., Tatebayashi, Y., Kijima, S., and Nishikawa, T. (1970). Subacute spongiform encephalopathy as a subtype of CreutzfeldtJakob's disease. Report of two cases. Psychiatria et Neurologia Japonica, 72, 669-690.

May, W. W. (1968). Creutzfeldt-Jakob disease. Actå̃ Neurologica Scandinavica, 44, 1-32.

Nelson, J. R., and Leffman, H. (1963). The human diffusely projecting system. Archives of Neurology (Chic.), 8 544-556.

Ohya, T., Martinez, A. J., Jabbour, J. T., Lemmi, H., and Duenas, D. A. (1974). Subacute sclerosing panence phalitis. Neurology (Minneap.), 24, 211-218.

Rayport, M. (1963). Electroencephalographic, corticographic and intracerebral potentials in two anatomically verified cases of Creutzfeldt-Jakob disease. Electroencephalography and Clinical Neurophysiology, 15, 922-931.

Roos, R., Gajdusek, D. C., and Gibbs, C. J. (1973). The clinical characteristics of transmissible CreutzfeldtJakob disease. Brain, 96, 1-20.

Scollo-Lavizarri, G. (1968). Continuous EEG and EMG recordings during night sleep in a case of subacute sclerosing leucoencephalitis. Electroencephalography and Clinical Neurophysiology, 25, 170-174.

Schlote, W. (1970). Subakute präsenile spongiforme Encephalopathie mit occipitalem Schwerpunkt und Rindenblindheit (Heidenhain-Syndrom). Archiv für Psychiatrie und Nervenkrankheiten, 213, 345-369.

Yuasa, R. (1969). The EEG findings of CreutzfeldtJakob disease. Clinical EEG, 11, 361-367. 